\title{
Articulating Value: Building a Culture of Assessment in Special Collections
}

IN OUR FAST-PACED, INFORMATION-RICH WORLD, special collections increasingly are looked to as a rich supply of unique, rare, and primary sources from which new knowledge, dynamic discovery, and intense learning experience can be generated. We attribute assumed value to these materials because they are rare, unique, and distinctive. Yet we increasingly face management environments in which long-held assumptions and practices are being challenged. We can no longer simply assume value. Instead, we must demonstrate impact.

\section{Shifting from Assumed Value to Evidence}

Cultural heritage organizations have long celebrated their distinguishing treasures. As Nicolas Barker notes in his introduction to the Association of Research Libraries' publication Celebrating Research, special collections and archives are "distinctive signifiers of excellence" and "trademarks" of their home institutions. ${ }^{1}$ Signaling our changing times, ARL's subsequent forum, "An Age of Discovery: Distinctive Collections in the Digital Age," further promoted this venerable positioning of special collections in the context of rapidly evolving technologies. ARL's Transforming Special Collections in the Digital Age Working Group continues to pursue "new models for assessing and optimizing the value of special collections in supporting institutional missions - in particular research, teaching, and learning — and against which to prioritize resource allocation and identify new directions."2 Concurrently, ARL has been exploring concrete means of demonstrating the value and return on investment of academic libraries through the Lib-Value project. ${ }^{3}$ ARL's struggle to move from celebrating its member libraries' treasures to proving their relevance and value is indicative of the challenge that institutions of many sizes and

1. Philip N. Cronenwett, Kevin Osborn, Samuel A. Streit, eds., Celebrating Research: Rare and Special Collections from the Membership of the Association of Research Libraries (Washington D.C.: Association of Research Libraries, 2007), 15.

2. "Transforming Special Collections in the Digital Age Working Group (charge)," February 11, 2010, available online at www.arl.org/bm doc/special-collections-working-group-charge-final.pdf.

3. "Lib-Value: Value, Outcomes, and Return on Investment of Academic Libraries," available online at http:/ /libvalue.cci.utk.edu/content/lib-value-project [accessed 12 August 2012].

${ }^{\star}$ Please note: URLs for all websites referenced herein were valid at the time of article submission. 
types that hold special collections and archives are presently facing. Documented evidence of impact has become particularly important as higher education models transition to global, participatory, online environments and the continuing economic crisis disrupts traditional bases of financial support for academic libraries.

It is becoming increasingly apparent that it is no longer enough to merely accumulate noteworthy collections. We have moved beyond an era in which simply acquiring and vaunting "treasures" is viewed as the hallmark of achievement. Every library, archives, and museum has collected rich accumulations of materials significant to its specific constituencies. And because so many organizations promote their riches through exhibition, outreach, publication, and digitization, all of our institutions appear "special." Indeed, it is by increasing these value-adding activities that libraries become more relevant to users. Value is a function of what institutions do with their rare and unique materials - how they connect them to the greater academic enterprise and to their users' development as scholars and lifelong learners. An organization's ability to effectively demonstrate that impact becomes critical to moving from "special" to "valuable."

Special collections librarians and archivists have long employed instinctual and rhetorical strategies for articulating value. Individual curators hold deep and broad expertise in their areas of collecting and expect to be trusted to know the impact their collections have on research based on their subject knowledge and years of experience. While such expertise should continue to be highly valued, a longerterm, sustainable future for collections and programs depends on more objective evidence of relevance and efficient, strategic, and judicious prioritization of effort. Curatorial instinct regarding the estimation of research value needs to be confirmed by hard data gathered through assessment.

Additionally, the budgets of cultural heritage institutions are static or decreasing. Supplemental sources of funding, such as private donors, granting agencies, foundations, and federal and state governments, are similarly pinched. Reduced resources combined with challenges to traditional academic models and the rapid transformations occurring in research, teaching, and learning require special collections and archives to demonstrate greater impact with more efficient methods than ever before. In their contribution to this issue of $R B M$, Joyce Chapman and Elizabeth Yakel have called attention to the "new inclination toward data-driven management" in library and higher education administration. ${ }^{4}$ They also observe that, as a whole, archives do not effectively use operational data to respond to this important trend.

4. Joyce Chapman and Elizabeth Yakel, "Data-Driven Management and Interoperable Metrics for Special Collections and Archives User Services,” RBM: A Journal of Rare Books, Manuscripts, and Cultural Heritage 13, no. 2 (Fall 2012): 129-151. 


\section{Articulating a Value Proposition}

While a shift from highlighting the intrinsic value of rare and unique collections to demonstrating impact based on access and use moves us toward a more grounded user- or customer-centered focus for special collections, in many cases we have yet to articulate a value proposition for special collections that is convincing enough to pry loose the resources needed to sustain and develop them. One method of framing a value proposition is based on an analysis of benefits versus costs, or outcomes as measured against inputs. ${ }^{5,6}$ The benefits or outcomes side of the equation requires persuasive methods of measuring and presenting access and use statistics as well as documenting impact on teaching, learning, and research-all in the context of and with demonstrated relevance to the larger institutional mission. Calculating the costs associated with effective discovery, access, and engagement is just as critical to constructing the proposition.

Essentially, we must ask: while our special collections and archives may have inherent value based on their rarity or uniqueness, how can we prove that they have relevance in today's research, teaching, and learning environments? In the context of the academic community, how do we measure the impact of our collections and services in ways that demonstrate meaningful contribution and success? What return on investment do our institutions obtain by devoting scarce resources to collecting, describing, preserving, and digitizing our collections? How are scholarship and learning transformed by our collections and services? How can we make data-driven decisions about where to direct limited resources and what we might need to stop doing in the process?

Further, the broader academic enterprise is increasingly collaborative and consortial. The roles played by special collections in teaching, learning, and research often extend beyond the local institution to influence its position among its peers. Increasingly, part of the value proposition should articulate how well we can help our parent organization partner with other institutions to build a combined body of

5. Several methods of determining value propositions in libraries are presented in the ACRL Value of Academic Libraries project at www.acrl.ala.org/value/ and in the report from the Association of College and Research Libraries, "Value of Academic Libraries: A Comprehensive Research Review and Report," researched by Megan Oakleaf (Chicago: Association of College and Research Libraries, 2010), especially 83-90.

6. A frequently cited source from business literature that presents approaches for formulating a value proposition is Cindy Barnes, Helen Blake, and David Pinder, Creating and Delivering Your Value Proposition: Managing Customer Experience for Profit (London and Philadelphia: Kogan Page, 2009). Barnes et al. stress that the process of creating a value proposition can be as important as the outcome, while noting that "a value proposition is not what you do. It is the value experience that you deliver" (7, emphasis in the original).

7. For further discussion of the need for special collections to cull low-value functions and services, see Merrilee Proffitt, "Something's Got to Give: What Can We Stop Doing in a Time of Reduced Resources?" RBM: A Journal of Rare Books, Manuscripts, and Cultural Heritage 12, no. 2 (Fall 2011): 89-91. 
resources. What do we offer the collective? What do we offer our home library as leverage with its collaborators? As our directors recalibrate their investment around collaborative action, where are our special collections positioned "as components of the academy's knowledge resources?"' Through the comprehensive assessment of our collections, our productivity, and the impact of our services, we can better articulate how we fit into larger opportunities for collaboration, partnership, and resource sharing.

By establishing the value proposition in these terms, we can gain the confidence of our funders. This type and level of trust is earned rather than assumed and is based on systematic, purposeful activity that employs evidence-based practice. The goal of assessment activities in this context is to show that we are good stewards, not just of our collections but of limited resources and, more importantly, the broader aspirations of our parent institution and the research community at large.

\section{Self-determination}

While measuring and achieving alignment with the broader institutional or even interinstitutional context is critical to securing support, special collections and archives should undertake assessment as much for our own immediate, local purposes. Because our rare and unique collections can have such a powerful draw on individual constituents, we often find ourselves capriciously reacting to the demands of a researcher or donor, a new funding opportunity, or new attention from an administrator. While such engagements can stimulate creative and mutually beneficial responses, a more methodical approach incorporating evidence-based decision making may prove to be more constructive in the long run.

As a community of professionals, we seek more satisfying ways to verify our impact, show success, and determine where to expend our limited resources. A 2010 ARL survey on engagement activities in special collections concluded that most respondents were exasperated by an inability to measure the impact of their work:

Institutions feel they are not able to quantify the success of their efforts, and this in turn limits the ability to compare activities within the institution or across institutions, to plan further outreach effectively, or to communicate the results of those outreach activities to the larger special collections community. ${ }^{9}$

8. Chris Avery, Sue Baughman, and Judy Ruttenberg, 21st-Century Collections: Calibration of Investment and Collaborative Action (Washington, D.C.: Association of College and Research Libraries, Issue Brief, March 10, 2012), available online at www.arl.org/bm doc/21stctfreport_11may12.pdf.

9. Adam Berenbak et al., SPEC Kit 317: Special Collections Engagement (Washington D.C.: Association of Research Libraries, August 2010), 16. 
A more broadly scoped survey conducted by OCLC Research similarly determined that "a lack of established metrics limits collecting, analyzing, and comparing statistics across the special collections community." ${ }^{10}$ The Archival Metrics project created assessment tools in response to a 2003 study that found the following:

the field recognizes a need for more robust and validated measures for both better internal administration and for benchmarking across peer institutions. Archivists and special collections curators expressed a great deal of frustration over current data collection and the inability to associate these data with real outcomes and impact measures. ${ }^{11}$

The Association of College and Research Libraries' Rare Books and Manuscripts Section (RBMS) has recognized this gap as well and has recently established a Metrics and Assessment Task Force to study current assessment practices and make recommendations for new community-based definitions and guidelines. ${ }^{12}$

Clearly, we recognize that our mechanisms for determining success are inconsistent at best and, in some cases, severely inadequate at providing data to inform decision making. As a community, we are craving methods to better evaluate our collections and services, prioritize actions to improve them, understand user and access needs, enhance discovery, and advance teaching and learning. The essays included in this issue of $R B M$ repeatedly affirm the value of assessment for establishing our own direction and priorities. Chapman and Yakel in particular point out that:

internally, in times of scarcer resources, special collections librarians and archivists are attempting to use data to manage their workflows more efficiently in order to accomplish such goals as maximizing access, increasing the impact of outreach, managing collections processing, exploring new staffing configurations, and making intelligent use of physical spaces. ${ }^{13}$

Even if we do not undertake assessment activities for the sake of our parent organizations, we should develop metrics and employ them in the interest of improving our own practices.

10. Jackie M. Dooley and Katherine Luce, Taking Our Pulse: The OCLC Research Survey of Special Collections and Archives (Dublin, Ohio: OCLC Research, 2010), 10, available online at www.oclc.org/research/ publications/library/2010/2010-11.pdf.

11. Elizabeth Yakel et al., "Developing Standardized Metrics: Towards Understanding the Impact of College and University Archives and Special Collections on Scholarship, Teaching, and Learning," Archival Metrics, available online at http://archivalmetrics.cms.si.umich.edu/node/16.

12. Association of College and Research Libraries, Rare Books and Manuscripts Section, "Metrics and Assessment Task Force," available online at www.rbms.info/committees/task_force/metrics_assessment/index.shtml.

13. Chapman and Yakel, "Data-Driven Management," 150. 


\section{Meaningful Assessment}

Assessment activities need to be not only grounded in shared best practices and tools to provide a basis for external benchmarking but also tailored to address local questions and concerns. While measuring our success against our peers may mark achievement toward a leadership position, ultimately such comparisons are most pertinent as points of reference toward continuous improvement. Collecting data for the sake of collecting data or in pursuit of improved rankings leads to aimless attachment to a set of numbers. Instead, we need to gather data that help us strengthen our collections, workflows, services, and their impact. As Anne Bahde and Heather Smedberg remind us in their contribution to this issue, "... with any assessment model, a clear understanding of what is being measured, and why, must be developed in order for the practice to become meaningful and effective." 14

Assessment that matters requires us to be clear on how we relate to the external drivers we need to address as well as the problems we are trying to solve internally. One special collections department might need to enhance its profile organizationally. Assessment that helps to measure alignment of strengths and activities against a larger mission and strategic plan may be in order. Another special collections library might have accumulated a processing backlog and can no longer sustain access based on the memory of its current staff. A collection-level survey may help get a handle on the magnitude of the challenge and lead to prioritization for resource requests. Another special collections unit might have to reduce hours or have the opportunity to hire. In this case, evaluation of data may indicate the most strategic reallocation of staffing.

Each of our special collections and archives may face different challenges but they share a common framework. As a field, we need an infrastructure of best practices and recommended tools that can then be applied thoughtfully and with intention. We must analyze carefully what we need to know, how we plan to use the data we accumulate, and what purposes it will serve. Otherwise, assessment activity risks not mattering at all.

Thoughtfulness requires time, knowledge, and effort. If we are going to undertake assessment or more of it, we need to make the commitment to doing it well. We need to research and employ existing methodologies so that we avoid duplicating efforts and wasting resources. We should also contribute to broader data-gathering efforts whenever possible. We need to identify data that already exist in our discovery tools and processes but could be better leveraged. We should seek advice and

14. Anne Bahde and Heather Smedberg, "Measuring the Magic: Assessment in the Special Collections and Archives Classroom," RBM: A Journal of Rare Books, Manuscripts, and Cultural Heritage 13, no. 2 (Fall 2012): 165 . 
partner with those who know more about assessment than we do, such as assessment librarians, our local school of management, and professional associations. In these ways, we can ensure that our assessment is as efficient, relevant, and collaborative as we want all our processes to be.

\section{A Path Forward}

This issue of RBM tackles the current state of assessment in special collections and archives head on. Within these pages, our colleagues evaluate a wide variety of methodologies and offer recommendations for how we might proceed individually and as a community.

\section{First, Know Your Collections}

Before we can articulate value, resource needs, or opportunities for impact, we need to know what we have. Are we relying on institutional memory or intuition to present our strengths? Or can we generate reports that can respond to any number of questions about the makeup of our collections? Library collection managers can provide reports about collection size, subject strengths, languages, and circulation. Such reports enable them to make data-driven decisions about allotment of space, realigning expenditures, and shared collecting initiatives. Special collections librarians and archivists must also find ways to generate data about our collections from a holistic, overall perspective to convey trends, expose patterns, and aid decision making.

Collections assessment serves as the foundation for all other efforts. Grant applications are stronger when collection strengths, physical condition, and usage can be quantifiably articulated. Acquisition decisions should be grounded in an understanding of gaps and strengths. The evaluation of workflow, use, instruction, and discoverability is richer in the context of collection data. As Martha Conway and Merrilee Proffitt note in their essay for this issue:

Armed with the array of information that collections assessment provides, many institutions find themselves empowered to tackle a variety of once-daunting tasks, to advocate for additional internal and external resources, and to work collaboratively toward common goals. ${ }^{15}$

Collections assessment is, therefore, a foundational activity that provides a springboard for action.

15. Martha O’Hara Conway and Merrilee Proffitt, "The Practice, Power, and Promise of Archival Collections Assessment," RBM: A Journal of Rare Books, Manuscripts, and Cultural Heritage 13, no. 2 (Fall 2012): 104 . 


\section{Process Improvement}

As budgets tighten and staffing levels change while expectations for productivity continue to rise, we must strive to make our processing, description, and digitization efforts as efficient and effective as possible. As our organizations focus on user success, we need to get more of our materials out there faster, for if our collections are not easily accessible they may as well not exist. We need to improve user convenience while pooling resources and avoiding redundancies. Yet we also need to ensure that more streamlined methods are applied appropriately to the collections in the context of their research value and their anticipated use. As we work, we need to determine what work provides value and what activity is unnecessary.

This requires us to know how much time we are spending on which activities and whether the output from those activities enhances discoverability or use. Analysis of workflow can establish expected baselines, expose duplicative effort, and analyze costs for the desired product, as Emily Gustainis has demonstrated through the Processing Metrics Collaborative project that she describes elsewhere in this issue. ${ }^{16}$ The outcomes of our effort should be tested against actual user methodology to determine usefulness. Such assessment can tell us more about what we can stop doing and enable us to determine whether investments in processing initiatives are yielding adequate returns.

\section{User-driven}

If collections assessment establishes a foundation for action and process assessment expedites access, then service assessment is core to understanding the true worth of our collections and the value we add to them. We cannot really begin to determine the return on our investment until we understand how scholars use the access tools we create. Fundamentally, we need to gather evidence of how our collections and services transform scholarship and generate new knowledge. As research-oriented, user-driven organizations, these are the most critical data we can gather.

On the one hand, as Chapman and Yakel suggest, we can better use the data we are already gathering through our daily processes. ${ }^{17}$ By improving our gathering and use of operational data, we can make better decisions about hours and staffing, identify outreach opportunities, and inform processing and digitizing priorities. On the other hand, deeper analysis of quality measures, such as those targeted by the user surveys included in the Archival Metrics toolkit, help us understand how well we are supporting our users' research needs. Strong and useful measures in this area are critical

16. Emily R. Novak Gustainis, "Processing Workflow Analysis for Special Collections: The Center for the History of Medicine, Francis A. Countway Library of Medicine as Case Study," RBM: A Journal of Rare Books, Manuscripts, and Cultural Heritage 13, no. 2 (Fall 2012): 114.

17. Chapman and Yakel, "Data-Driven Management." 
to articulating the value proposition of special collections and determining where we can make the best investments of our staff effort and other resources.

\section{Impact in the Classroom}

Similarly, as our libraries increasingly pursue opportunities to improve students' knowledge-building skills, impact undergraduate research, and partner with instructors and faculty to advance teaching, special collections must demonstrate how we contribute to those goals. Our research rooms are laboratories for exploration, discovery, and insight. Special collections librarians and archivists are increasingly present in the classroom and other student pathways. As we engage learners and their instructors, we need mechanisms for determining the impact of our efforts. We need to know and be able to demonstrate how well we are helping students to become better scholars, connect with creativity, and develop lifelong learning habits.

We can begin by partnering with faculty not only to establish curricular content and learning outcomes but also to determine how to measure the contribution of special collections to those outcomes. As Bahde and Smedberg point out, we should be able to adapt assessment methods already used in the broader academic and library contexts to gauge our success; ${ }^{18}$ doing so might mean working closely with instruction librarians or campus centers that aim to improve teaching. Determining the value of educational experience is a dynamic, evolving area, and we must be prepared with appropriate assessment strategies so that we can improve instructional design, adjust technique to learning outcomes, and modify future teaching performance.

\section{Ease of Use}

As we surface our collections, expedite access, increase usage, and enhance research and learning, we invest heavily in online discovery and use environments for our collections. Special collections and archives are spending significant resources on digitization and on creating EAD-encoded finding aids, online exhibits, mobile apps, and social networking spaces. Research and learning are increasingly mediated online. How do we know that our collections are discoverable, understandable, and usable in the online environment? How can we find out whether they are unknown or impenetrable, and how can we make access easier and more intuitive?

The investment we make in creating online tools must be accompanied by a commitment to assess the success these offerings have on virtual access, online research, self-service, and participatory learning. The utility of our finding aids, design of our interfaces, and relevance of our social networking determine our 
success in reaching through the information explosion to connect our collections to the users who value them. As Rachael Hu points out in her contribution to this issue of $R B M$, usability studies are critical to ensuring that our efforts to increase online access are well spent, to identifying additional skills or tools needed to improve access, and to demonstrating to resource allocators the virtual reach of our services and collections.

\section{Moving Forward, Purposefully}

The opportunities and needs for assessment activities related to special collections and archives can seem overwhelming given the lack of established metrics and methodologies. The essays in this issue of $R B M$ outline pathways and approaches to conducting meaningful assessment in five broad areas of special collections and archival management. As we extend and strengthen our culture of assessment in special collections and archives, here are a few things to keep in mind:

- Identify what it is that you want to know. Recognize why you are gathering information and how you plan to use it. Such understanding will ensure that the data you are gathering is useful.

- Begin strategically. Undertake assessment in an area that has direct relevancy to a problem you are trying to solve or a message you are trying to share.

- Do not reinvent the wheel. Whenever possible, use tools and methods that have been successful elsewhere and adopt prevailing frameworks. Analyze data you are already gathering as part of existing processes.

- Learn how to effectively analyze the data gathered and tap into existing expertise to show you how.

- Use assessment findings to implement change. Otherwise, data gathering and analysis simply drain resources.

- Coordinated effort is more efficient than individual exertion and results in comparable data. As a field, we should establish best practices and shared tools, aligning them with current initiatives valued by our partner and parent organizations.

In today's environment of reduced resources, outcomes-based decision making, and increased expectations and possibilities for enhancing value for our users, special collections and archives must embrace a culture of assessment to demonstrate our success, articulate our needs, and evaluate our opportunities. We must articulate our relevance to our constituencies, our funders, and ourselves. We must ground our advocacy and resource allocation in evidence provided by verifiable analysis. We must prioritize activity that has the greatest impact on mission. If the 
future of academic and research libraries is tied closely to their unique and distinctive resources, a culture of assessment focused on creating and communicating value provides direction, verifies strategies, and indicates improvements needed to effectively leverage our special collections for greatest impact.

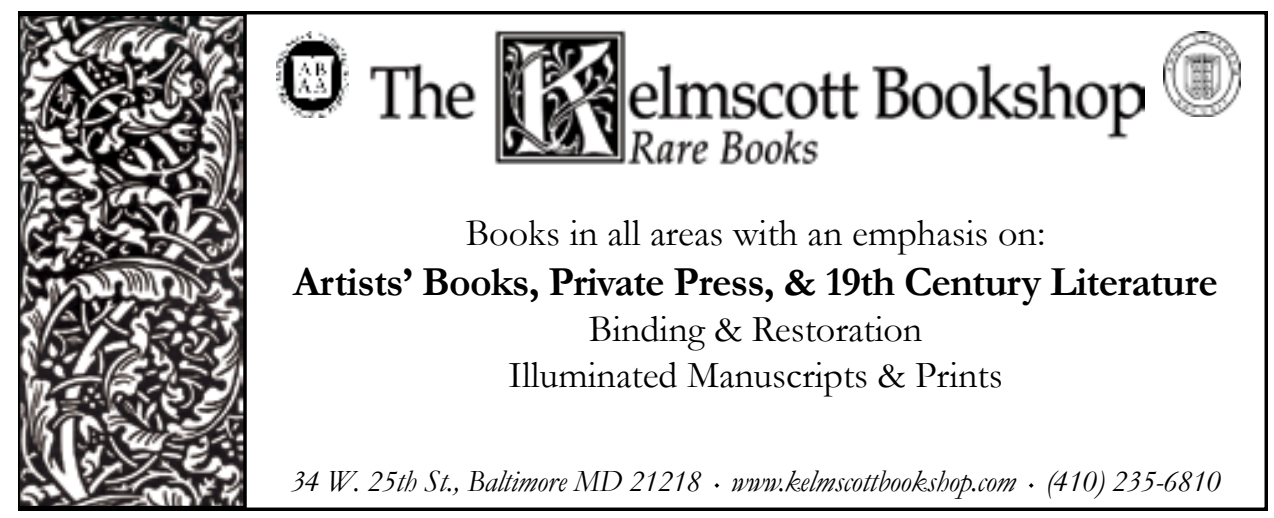

